\title{
A new target for differentiation therapy in AML
}

\author{
Cell Research (2017) 27:9-10. doi:10.1038/cr.2016.130; published online 11 November 2016
}

Despite major advances in understanding the genetics and epigenetics of acute myelogenous leukemia, there is still a great need to develop more specific and effective therapies. High throughput approaches involving either genetic approaches or small molecule inhibitor screens are beginning to identify promising new therapeutic targets.

One approach to develop new acute myelogenous leukemia (AML) therapies has been to design mechanismbased inhibitors. This has led to a number of new therapeutic agents entering clinical trials, including inhibitors of the DOT1L methyltransferase, BRD4 and IDH1/IDH2 [1]. This approach requires knowledge of the driver oncogenic mutation and is limited to specific genetic subtypes of AML. A further challenge is that other cooperating oncogenes may confer chemotherapy resistance.

An alternative approach is to screen for agents that overcome the block to myeloid differentiation seen in AML. The most notable success was the discovery that trans retinoic acid (ATRA) induces differentiation of promyelocytic cell lines. This has led to the successful use of ATRA for patients with APL [2]. But how can similar agents to overcome differentiation block for the other $90 \%$ of AML cases be identified?

Sykes et al. [3] recently reported a powerful approach that should be broadly applicable to discovering new differentiation inducers in a wide range of AML cases. The authors noted that the homeobox-containing transcription factor HOXA9 is overexpressed in more than $50 \%$ of AML cases, including leukemias with MLL rearrangements,
NUP98 translocations, NPM1 mutations and CDX2/CDX4 overexpression. Furthermore, this deregulation is important for leukemogenesis [4]. The authors generated an inducible model of myeloid differentiation arrest by expressing an estrogen receptorHoxA9 (ER-Hoxa9) fusion protein in murine bone marrow cells. Addition of $\beta$-estradiol (E2) to ER-Hoxa9-expressing cells induces the translocation of ER-HoxA9 into the nucleus thereby blocking myeloid differentiation. These immortalized cells were arrested at the granulocyte-macrophage progenitor (GMP) stage, but undergo synchronous and terminal neutrophil differentiation upon E2 withdrawal. To facilitate the screening of small molecules stimulating myeloid differentiation, Sykes et al. generated a Lys-GFP-ER-HOXA9 cell line with the inducible HOXA9 using knockin mice, in which GFP is inserted into the lysozyme locus. Lysozyme is only expressed in mature granulocytes, so that GFP expression serves as a reporter for screening compounds that induce differentiation (Figure 1). A library of 330000 molecules was screened and of these, 12 induced myeloid differentiation. The activity of these was further tested in other AML lines and two with distinct chemical scaffolds ( $\mathrm{C} 03$ and C07) were selected for optimization because of their activity in both murine and human AML cells.

The next challenge was to identify the protein target of the small molecules responsible for differentiating activity. The authors took the approach of growing the mouse and human cell lines for a long period in either $\mathrm{C} 03$ or $\mathrm{C} 07$ and analyzing the gene profiles of the drug- resistant cells by RNA-Seq. The resistant cells showed remarkably similar expression profiles, with up regulation of only eight shared genes that turned out to be located within a $100 \mathrm{~Kb}$ region of the long arm of human chromosome 16 or murine chromosome 8 . One of these genes encoded dihydroorotate dehydrogenase (DHODH), a critical enzyme in uridine synthesis that catalyzes the conversion of dihydroorotate to orotate (Figure 1). Intriguingly, in addition to $\mathrm{C} 03$ and $\mathrm{C} 07$, all but one of the other 12 compounds identified in the small molecule screening were also found to be DHODH inhibitors.

These results strongly suggest that DHODH, and its downstream metabolic product uridine, regulate myeloid differentiation. This possibility is further supported by the finding that uridine can rescue the blockage of cell differentiation induced by DHODH inhibitors and that inhibiting orotidine $5^{\prime}$-phosphate decarboxylase, another essential enzyme regulating pyrimidine synthesis, causes a similar block in myeloid differentiation as that induced by the DHODH inhibitors.

The authors developed a lead compound ML390, derived from CO7, but it was found to be poorly bioavailable. Instead, for in vivo experiments, the authors chose a potent and selective inhibitor of DHODH, brequinar sodium (BRQ), which had been tested clinically for other tumors, but not leukemia. Encouragingly, BRQ treatment significantly induced myeloid differentiation, delayed the disease development and reduced the burden of leukemia-initiating cells in the various AML mouse models, including human cell line xenografts, 


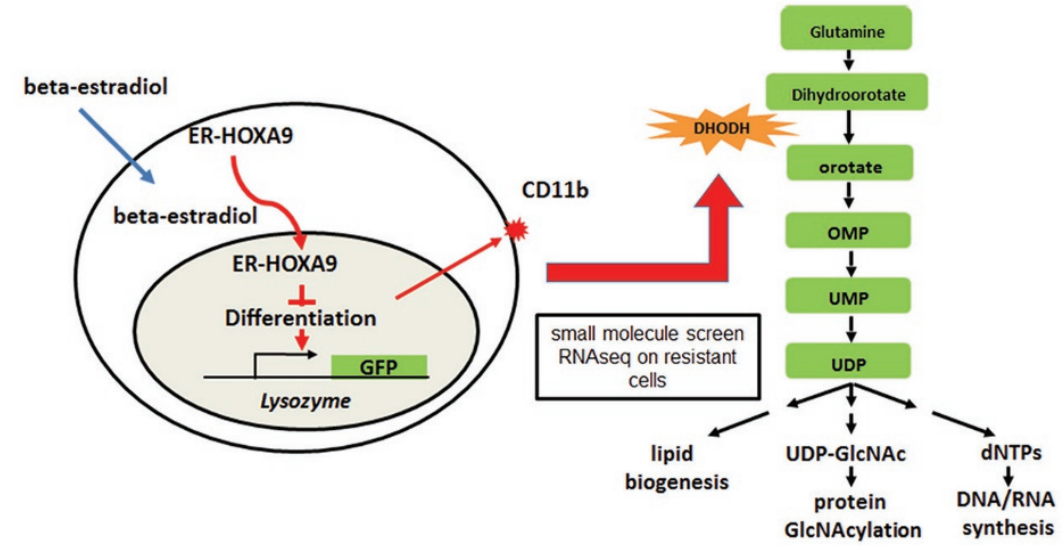

Figure 1 Identification of DHODH as a target for differentiation therapy. Myeloid cells were conditionally immortalized by expression of estrogen receptor-HoxA9 (ER-HOXA9) fusion protein in bone marrow cells in which GFP is inserted into the lysozyme locus. Removal of $\beta$-estradiol (E2) causes ER-HOXA9 to remain in the cytoplasm resulting in myeloid differentiation and expression of GFP and granulocyte markers such as CD11b. Screening 330000 compounds led to the identification of small molecules promoting myeloid differentiation. Ultimately the target of the inhibitors was identified as $\mathrm{DHODH}$, a key regulator of pyrimidine biosynthesis.

patient-derived xenografts, and syngeneic mouse models caused by a variety of genetic alterations.

The experimental approach of Sykes et al. is a powerful example of how high throughput strategies can be employed to identify new targets in AML and other malignancies. In particular the results show considerable promise for DHODH inhibitors, which have previously been used for treatment of other cancers, such as melanoma as well as rheumatoid arthritis and multiple sclerosis, for able to DHODH depletion. teins regulating differentiation. There is no shortage of candidates involved. Proteins known to be regulated by GlcNAcylation include RNA polymerase II, transcription factors such as $\mathrm{c}-\mathrm{MYC}$, and epigenetic regulators such as the Polycomb protein RING1B [6]. Aside from further delineating this mechanism, it will be important to test how broadly DHODH inhibition is effective given the heterogeneity of human leukemias and also what drugs will be most successful in combination with DHODH inhibition for complete eradication of leukemia.

Peilin $\mathrm{Ma}^{1}$, Weihua Song ${ }^{1}$, Jay L Hess ${ }^{1}$

${ }^{1}$ Department of Pathology and Laboratory Medicine, Indiana University School of Medicine, 340 West 10th Street, Fairbanks 6200, Indianapolis, IN 46202-3082, USA

Correspondence: Jay L Hess

E-mail: jayhess@iu.edu

\section{References} approach, a CRISPR “dropout” screen, Tzelepis et al. [5] found that a variety of AML cell lines are selectively vulner-

While the precise targets affected by $\mathrm{DHODH}$ inhibition remain to be identified, since DHODH is required for UDP-GlcNAc formation and DHODH inhibition reduced protein $O$-linked $N$-glycosylation in the AML cells, the inhibitor effects may be mediated through reduced glycosylation of pro-
1 Stein EM, Tallman MS. Blood 2016; 127:7178.

2 Lo-Coco F, Avvisati G, Vignetti M, et al. $N$ Engl J Med 2013; 369:111-121.

3 Sykes DB, Kfoury YS, Mercier FE, et al. Cell 2016; 167:171-186.

4 Collins CT, Hess JL. Oncogene 2016; 35:1090-1098.

5 Tzelepis K, Koike-Yusa H, De Braekeleer E, et al. Cell Rep 2016; 17:1193-1205. 289:34440-34448.
6 Lewis BA, Hanover JA. J Biol Chem 2014; 